\title{
Shape recognition with point clouds in rebars
}

\author{
Kosei Ishida ${ }^{1}$, Naruo Kano ${ }^{1}$, and Kenji Kimoto ${ }^{2 *}$ \\ ${ }^{1}$ Department of Architecture, Waseda University, Tokyo, Japan \\ ${ }^{2}$ College of Engineering, Shibaura Institute of Technology, Tokyo, Japan \\ *Corresponding author (k_ishida@y.aoni.waseda.jp)
}

\begin{abstract}
Purpose In this paper, the authors describe the methods of inspecting the quality of reinforced concrete structure using point-clouds data acquired from a 3D-laser scanner. A 3D-laser scanner is an outstanding device to analyze a realworld object and to collect digital data on its shape. Inspections of the quality of reinforced concrete structure using point clouds are required for novel methods which count the quantity of rebar material and to check the space of each rebar. Method To inspect with the use of 3Dpoint clouds, we developed a method of 3D-point-clouds recognition of the rebars elements. In this paper the authors show three methods to analyze point clouds collected in rebars of building structures. Firstly, the authors developed a method of noise reduction to make a clear distinction between object surface points and noise points; this is important because point clouds in rebars have much noise that can disturb subsequent analysis. Secondly, the authors developed a method of abstracting point clouds on reinforcement bars. Thirdly, the authors developed a method dividing point clouds on reinforcement bars into hoops and wall horizontal reinforcement bars. The authors scanned reinforced bars of columns and walls at a construction site of an apartment and then applied the three methods to analyze the point clouds data. Results \& discussion In this experiment, our methods were able to identify 3D point clouds as main bars, horizontal bars, and hoops. We were able to measure the object from 3D-point-clouds data at any time as well as being able to develop an automatic inspection system.
\end{abstract}

Keywords: quality control, 3D laser scanner, 3DCAD, point cloud, reinforcement work

\section{INTRODUCTION}

A 3D scanner is an outstanding device to collect digital data as 3D point clouds and analyze a realworld object. These digital data facilitate the measurement at any time because point clouds record the shape of an object.

There have been many studies ${ }^{1}$ of $3 D$ scanner in mechanical engineering and civil engineering. In recent years, there have also been similar studies ${ }^{2}$ in building engineering.

In construction engineering, many researchers have adapted $3 d$ scanner to create $3 D$ building models and have developed the method $^{3}$ of the shape recognition of $3 \mathrm{D}$ point clouds.

However, many studies in construction engineering have dealt with point clouds data processing in order to identify semantic feature, such as walls, floors, celling, windows or rooms.

The authors have developed a system to inspect the quality of reinforced concrete structure using point clouds data acquired with 3D laser scanner, focusing the shape recognition of 3D point clouds in reinforcing bars.

The building regulation in Japan has defined the rebar arrangement for resistance to collapse. The structure engineer determines the diameters of bars, an arrangement of vertical bars, and the size and spacing of columns ties.

Therefore, the construction workers have to confirm the rebar arrangement. But, rebar inspection is hard work and time-consuming work because many confirming spots exist in a construction site.

Thus, the authors have developed the methods of inspecting rebar arrangement.

In this paper, the authors show the three methodologies to analyze point clouds of reinforcing bars in construction sites: noise reduction, abstraction of point clouds of reinforcing bars, and shape recognition of vertical bars, columns ties, and horizontal bars of walls.

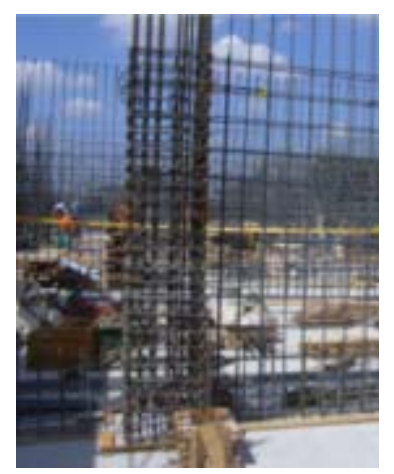

Fig.1 Reinforcing bars in Japan

\section{THE METHODS OF ANALYZING POINT CLOUDS}

\section{Point clouds of steel reinforcing bars}

Steel reinforcing bars ("rebar") for concrete construction is commonly used as a tensioning device in reinforced concrete. Rebar arrangement differs from columns, beams or walls. Columns contain two types of reinforcing bars: Vertical bars with a largerdiameter share the compressive loads with the concrete and resist the tensile stresses. Ties with a smaller-diameter are wrapped around the vertical bars. The ties may be either of two types: column 
ties or column spirals. In Japan, column ties are typical ties. Vertical bars and column ties are often a rectangular arrangement.

The authors scanned the reinforcing bars of the column and the wall using $3 \mathrm{~d}$ laser scanner (Figure 2) and obtained the point clouds of reinforcing bars (Figure 3). Point clouds of reinforcing bars record arrangement of reinforcing bars, but they often include many noises (Figure 4). Point clouds of vertical bars are similarly to cylinder because vertical bars have large-diameter bars (Figure 5). On the other hand, point clouds of column ties and bars in walls are linear, because column ties and bars in walls use bars with a small-diameter (Figure 5 and Figure 6).

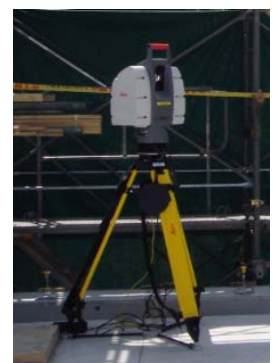

Fig.2 3D scanner

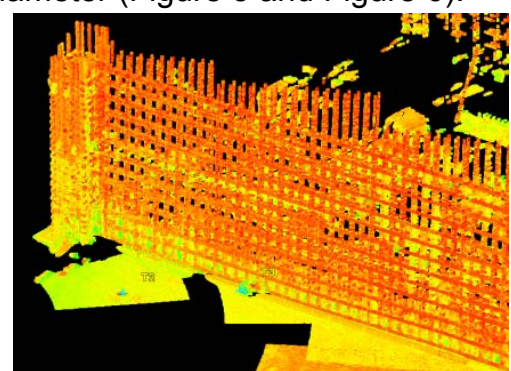

Fig.3 Point clouds of reinforcing bars

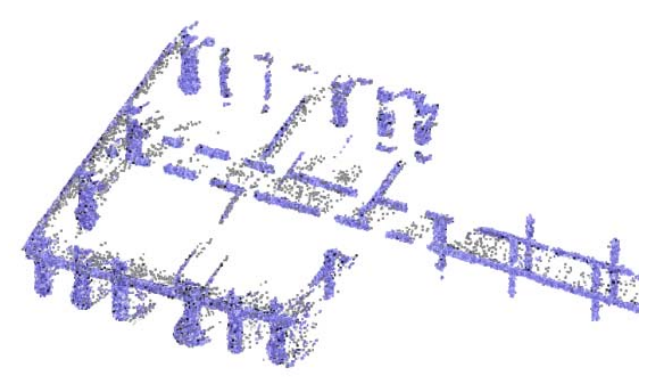

Fig. 4 Point clouds of a column ties including much noise.

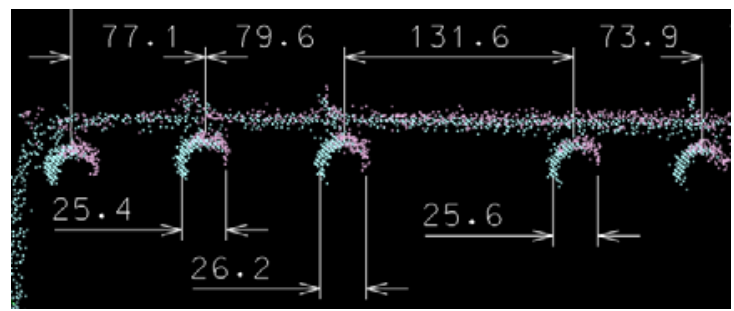

Fig.5 Point clouds of vertical bars and column ties.

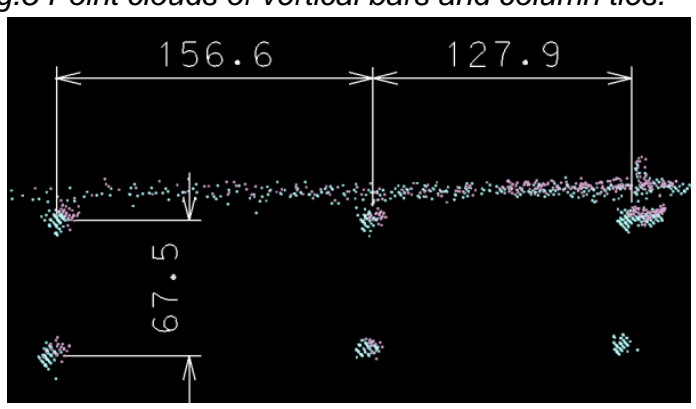

Fig. 6 Point clouds of vertical bars and horizontal bars in a wall.

\section{Steps of automatic inspection system}

Acquired raw point clouds data have a lot of noises, and do not provide the accurate semantic information of the structure. Therefore, the authors developed the method that extracts semantic information about rebar inspection from raw point clouds. The automatic rebar inspection system consists of the following five steps:

(a) Noise reduction makes a clear distinction between object surface points and noise points

(b) Multiple scans from different directions are brought in a common coordinate system, which is usually called "registration", and then merged into the complete point clouds data

(c) Point clouds of reinforcing bars are abstracted from the merged point clouds

(d) Point clouds are decomposed into three pieces: the linear point clouds which are vertical and parallel to the wall baseline, as well as point clouds of which direction is parallel to the floor and is at right angles with the wall baseline

(e) Pieces are assembled into characteristic configuration at a higher semantic level, e.g., vertical bars of columns, columns ties, vertical bars of walls and horizontal bars of walls

\section{Noise reduction}

When 3D laser scanner is applied to reinforcing bars, the point clouds contains many noises.

A point becomes a noise when the laser spot is distributed on several surface parts with different distances from the scanner (A point "Pi" in Fig. 7 represents a noise). Therefore, there are two characteristics: a point of noise has a longer mutual distance between the two points, and the density of point clouds is thin.

Noise reduction which is based on mutual distance sorts out a noise by an interval between two points. Using 3D scanner, the authors control the scanning interval on objects (bars) smaller than $4 \mathrm{~mm}$. Therefore, if an interval between the points is larger than the scanning interval, the point should be a noise.

$$
\begin{aligned}
& \mathbf{d}_{i}=\sqrt{\left(x_{1}-x_{1-1}\right)^{2}+\left(y_{1}-y_{1-1}\right)^{2}+\left(z-z_{l-1}\right)^{2}} \\
& \quad \mathbf{d}_{\mathbf{i}}>\boldsymbol{\alpha} \mathbf{D} \text { and } \quad \mathbf{d}_{i+1}>\boldsymbol{\alpha} \mathbf{D} \quad \ldots[1] \\
& D \text { : D is a set point interval of } 3 \mathrm{D} \text { scanner } \\
& \alpha \text { : Noise recognition coefficient ( about 1.5 2.0) }
\end{aligned}
$$

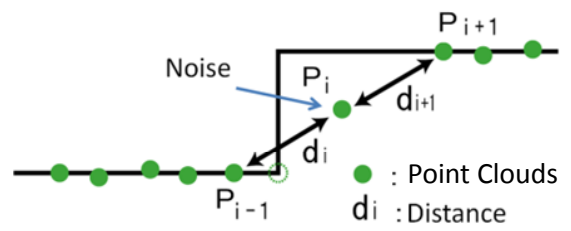

Fig.7. Noise characteristic 
When a point of [i] is $\mathrm{P}(\mathrm{i})$, "di" represents the distance between $\mathrm{Pi}$ and $\mathrm{P}(\mathrm{i}+1)$. The scanning interval is $\mathrm{D}$. The distance "di" is obtained from the following equation. If formula [1] is satisfied, $\mathrm{Pi}$ should be a noise.

Additional noise reduction is based on the density of point clouds. A point on the surface of an object is usually in a large number of point clouds per unit volume of a cube. By contrast, a noise point is in a small number of point clouds. Thus, it is possible that noise is detected by the density of point clouds.

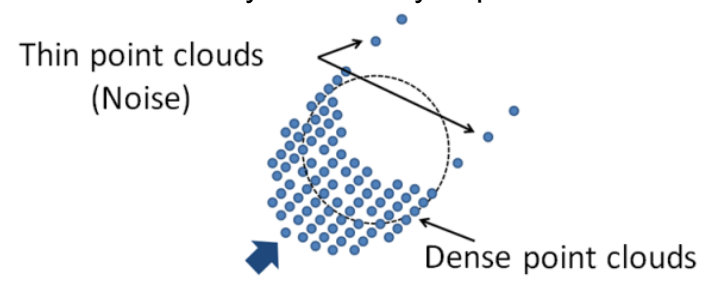

The direction of laser

Fig.8. Noise reduction based on density

\section{Abstracting point clouds of reinforcing bars $\mathrm{fr}$ om raw point clouds}

The method of abstracting point clouds of reinforci ng bars

Point clouds of reinforcing bars have a complicated shape. Therefore the point clouds of reinforcing bars cannot be distinguished directly from raw point clouds by using the mathematical methods such as least-square method.

Hence, the authors have developed a method of abstracting point clouds of reinforcing bars from raw point clouds.

The method of abstracting point clouds of reinforci ng bars in three dimensional spaces

Point clouds of reinforcing bars are not a primitive shape such as plane, sphere and cylinder.

Although point clouds of a primitive shape don't include point clouds of reinforcing bars, point clouds of non-primitive shape include more much point clouds of reinforcing bars than raw point clouds data. Therefore, if point clouds of a primitive shape are removed from raw data, point clouds including reinforcing bars remain.

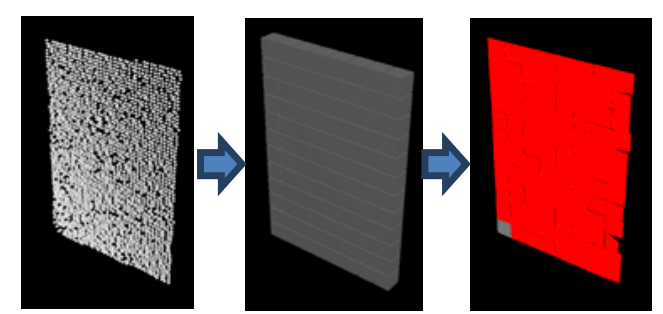

Fig. 9 Point clouds put into voxels and then replacing planes using least-square method

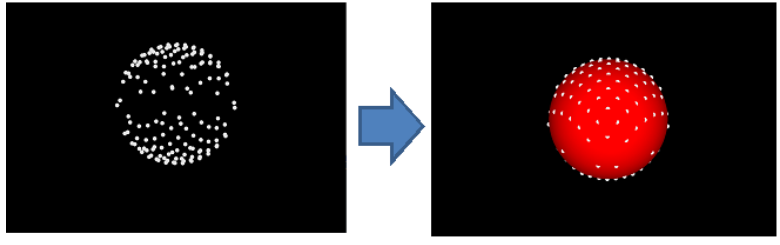

Fig.10 Point clouds of sphere replacing a sphere model using least-square method

Raw point clouds are put into voxels which represent a value on a regular grid in a three dimensional space (Figure.9).

Next, the point clouds in a voxel are fitted into a sphere and plane by the least-squares method. If the sum of the squares of the errors is less than tolerance, the point clouds are fitted into a sphere (or a plane) model and then the point clouds are removed from raw data (Figure 9 and 10).

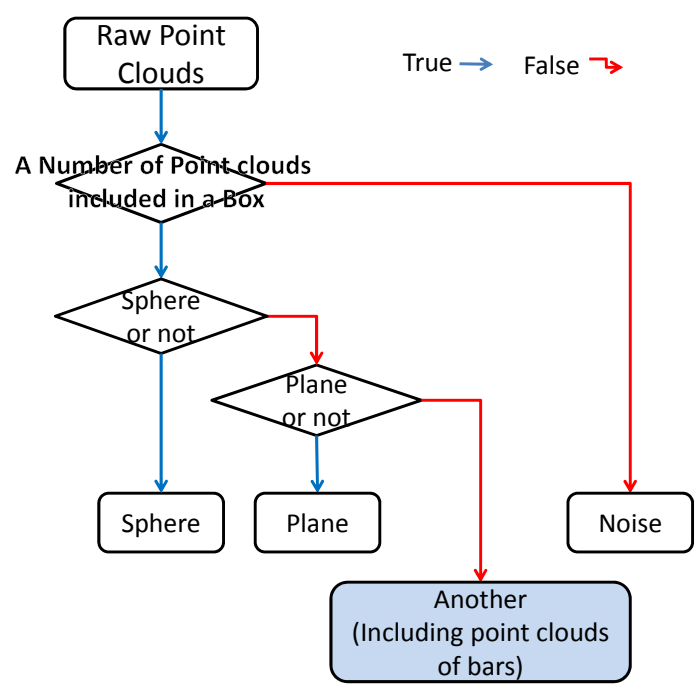

Fig.11 An example of the flow diagram dealt with shape recognition in three-dimensional spaces.

Remaining point clouds, which include point clouds of reinforcing bars, go on to next steps for abstracting point clouds on reinforcing bars in a cross section.

Steps of abstracting point clouds on reinforcing bar in a cross section

The method of abstracting point clouds on reinforcing bars in a cross section are developed by the authors consisted of the following three steps:

(a) The point clouds are sliced in horizontal 2D cross sections (Figure.12)

(b) In a cross section, point clouds is grouped by taking account of the continuity between pixel blocks (Figure.13 and 14)

(c) The system recognizes the shape in each cluster of point clouds and then distinguishes point clouds on reinforcing bars (Figure.15) 


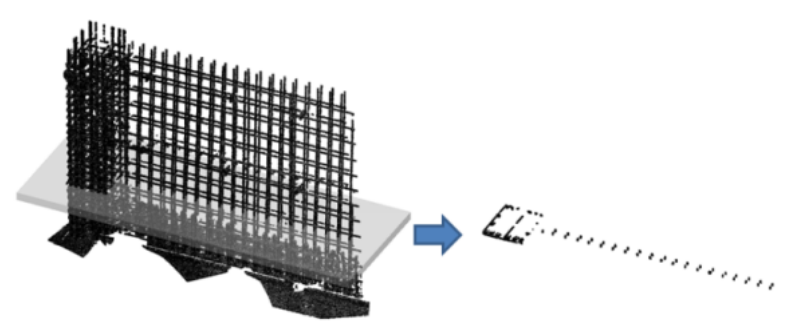

Fig.12. Image of point clouds sliced in a horizontal cross section

Replacing point clouds with pixel blocks and maki ng a group of neighboring point clouds

The sliced point clouds are analyzed with pixel blocks. The pixel block is a $5 \mathrm{~mm}$ square. If a point is located in a pixel block, a point replaces a pixel block as shown in Figure.13.

After replacing all point clouds, pixel blocks are grouped by taking account of the continuity between pixel blocks. Neighboring pixel blocks are four neighboring squares (Figure.14).

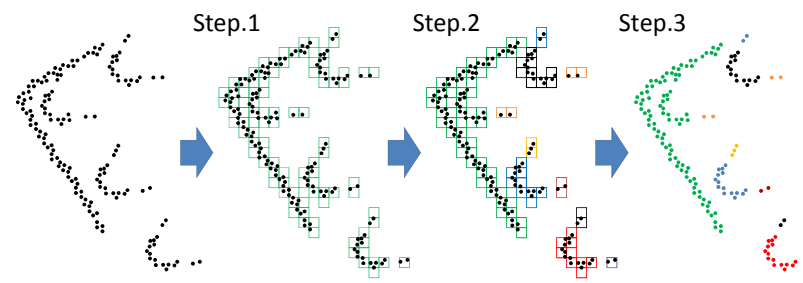

Fig.13. Image of pixel blocks and clusters

Neighboring Squares

Core Square

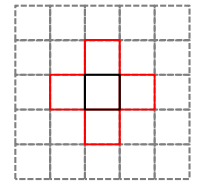

Four Neighboring Squares

Fig.14 Kinds of neighboring pixel blocks

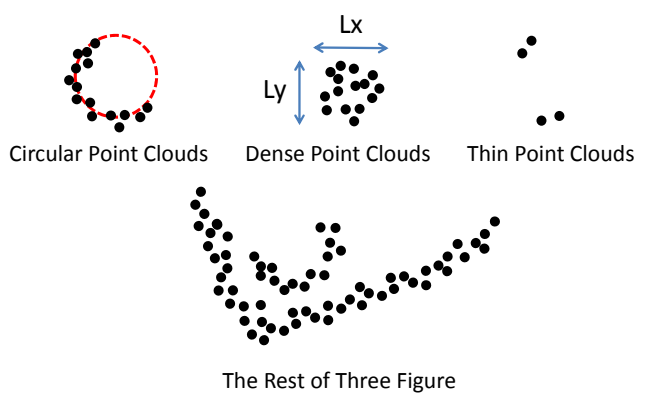

Fig.15. Classification of shapes in a cross section

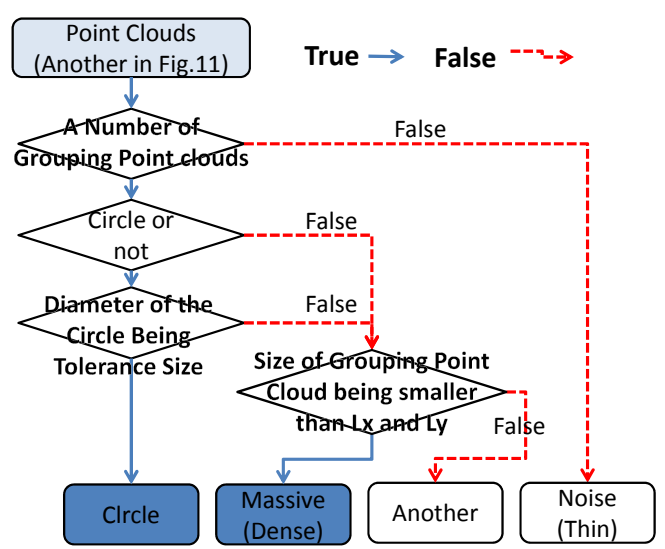

Fig.16 An example of the flow diagram dealt with shape recognition in two-dimensional plane (in a cross section).

Shape recognition of grouped point clouds in a cro ss section

The system then recognizes the shape in each cluster of point clouds. The classification of shapes in a cross section is shown in Figure.15.

When point clouds on vertical bars are sliced in a horizontal cross section, the density of point clouds on the cross section is high. On the other hand, when point clouds on horizontal bars are sliced in a horizontal cross section, point clouds in the cross section appear in a line. Thin point clouds are noise or unrecognizable points. Point clouds on the floor appear as a plane. Therefore, dense point clouds and circular point clouds should be point clouds on a rebar.

Method of making a distinction between vertical bars and the others

Because the shape recognition in a cross section makes some mistakes, the authors also developed a method making a distinction between vertical bars and the others.

The shape of bars is normally a smooth curve. Therefore, if point clouds are on a vertical rebar, clusters of dense point clouds could tie them in a row (Figure.17).

By contrast, if clusters are not on a vertical rebar, clusters of point clouds cannot tie them in a row.

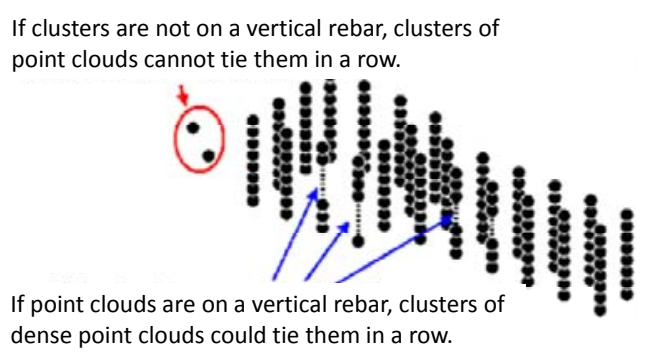

Fig.17. Abstracting point clouds on a vertical rebar 


\section{Decomposing point clouds into the linear pieces}

The reinforcing bars of walls and columns are arranged in a specific direction. The direction of wall reinforcing bars is either horizontal or vertical.

A column ties is a rectangle and the rectangle is divided into two directions at the corners.

Therefore, if point clouds of column ties are divided at the corners, they are decomposed into linear point clouds.

The system is able to slice point clouds not only in a horizontal plane but also in a plane which is at right angles to the wall baseline (Figure.18) as well as in a plane which is at right angles to both of the two planes.

On each plane, the system distinguishes point clouds on the vertical bars which are at right angles to the plane from the other point clouds (Figure.19). Finally, all point clouds of reinforcing bars are decomposed into linear point clouds.

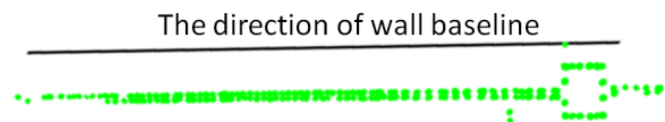

Fig.18 The direction of baseline

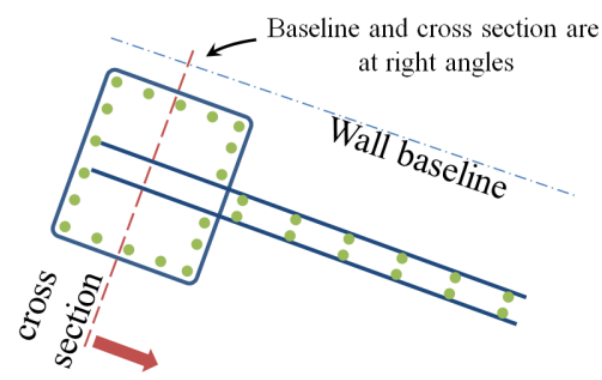

Fig.19 Cross section and wall baseline

\section{Assembling pieces of point clouds into colum ns ties}

After point clouds are decomposed into pieces, pieces of point clouds are assembled into column ties (Figure.21).

Decomposing point clouds into pieces

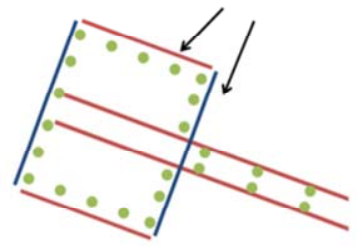

Assembling point clouds into column bars

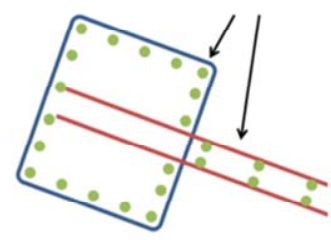

Fig.20 Decomposing into pieces and assembling the pieces into column ties

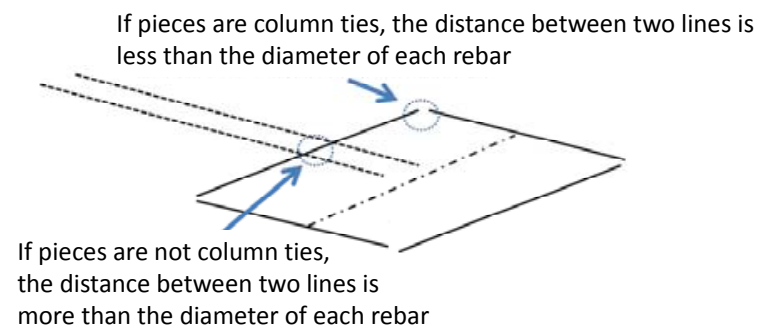

Fig.21 Geometrical features of a hoop

\section{APPLICATION OF THE SYSTEM TO POINT CLOUDS OF REINFORCING BARS}

\section{Point clouds of reinforcing bars obtained by la ser scanning}

The authors scanned reinforced bars of columns and walls at a construction site of an apartment house (Figure .22).

To evaluate the system capability of inspection, the authors have applied the system to the point clouds of reinforcing bars.

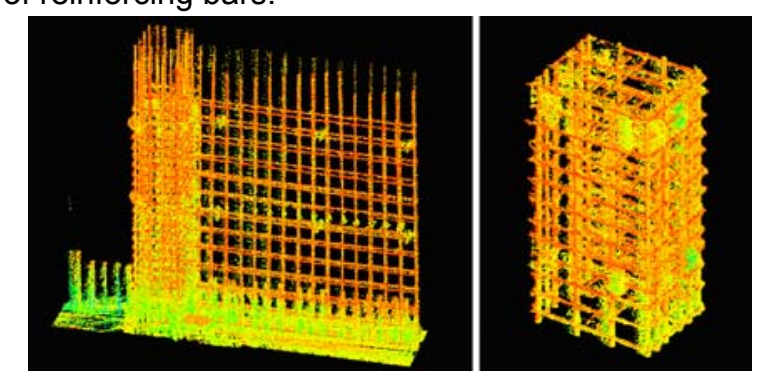

Fig.22. Point clouds on reinforcing bars of a column and a wall

\section{Noise reduction}

Fig.23 shows a result of noise reduction.

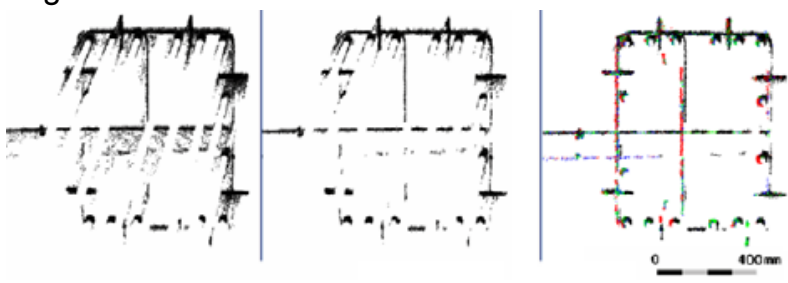

A. Acquired data B. Mutual distance C. Density Fig.23 Noise Reduction

Fig.23.A depicts the acquired point cloud data.

Fig.23.B shows a result of noise reduction based on the mutual distance. Fig.23.C shows a result of noise reduction based on the density. Almost all noises have been removed.

The result of abstracting point clouds of reinforcing bars in three dimensional spaces

Fig.24 shows a result of abstracting point clouds of reinforcing bars in three dimensional spaces.

Red point clouds are on a plane. Black point clouds are thin point clouds. Blue point clouds are remaining point clouds which include point clouds of rein- 
forcing bars.

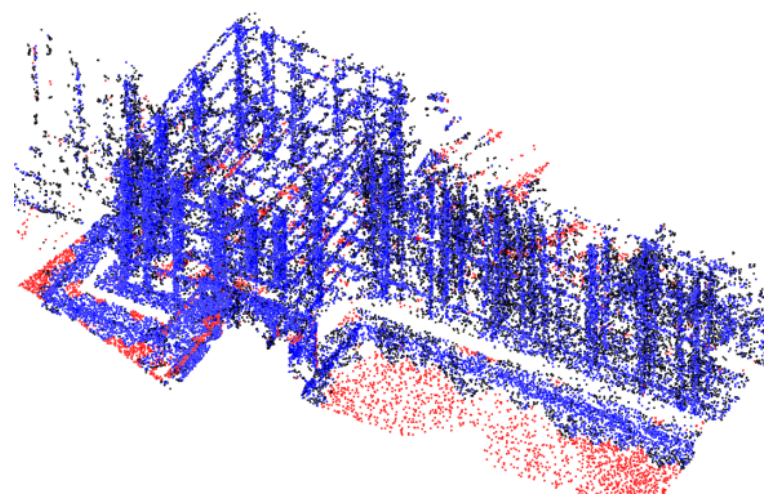

Fig.24 The result of abstracting point clouds of reinforcing bars in three dimensional spaces

\section{Distinction between vertical bars and the oth- ers}

After making a group of neighboring point clouds, the system recognized shapes in each cluster of point clouds and then abstracted point clouds on bars. Figure. 26 shows a result of shape recognition.

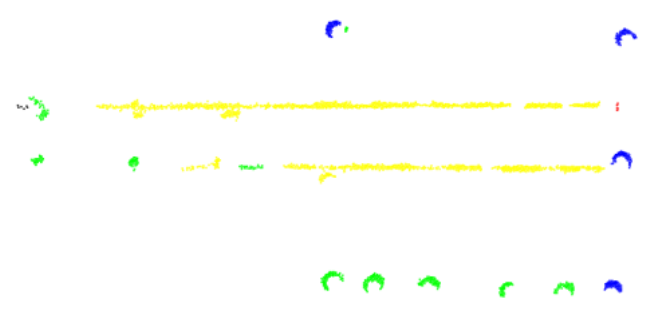

Fig.25 Example of shape recognition in a cross section (Blue: Circular, Green: Massive, Yellow: Another)

These point clouds are not on a vertical rebar,

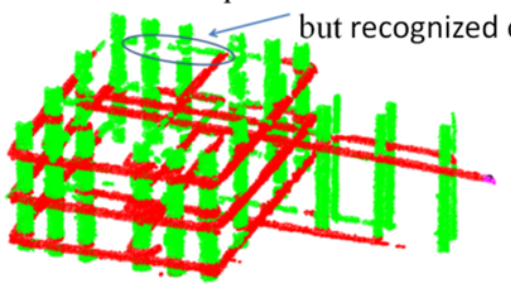

Fig.26 A result of Shape Recognition

Despite the fact that point clouds are not on a vertical rebar, a few point clouds are recognized on a vertical rebar because of the failure of shape recognition. However, Figure.27 shows a successful abstraction of point clouds by tying them in a row.

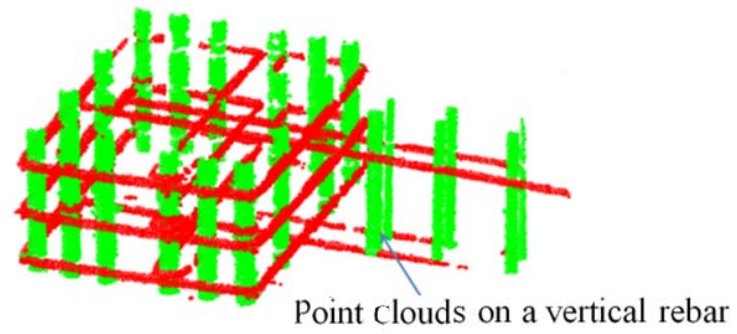

Fig.27 The result of abstracting point clouds in a smooth curve

\section{Decomposing point clouds into pieces}

Fig.28 shows a result of dividing point clouds of reinforcing bars at the corners and decomposing point clouds of reinforcing bars into linear point clouds. The directions of the linear point clouds are vertical and parallel to the wall baseline, as well as parallel to the floor and at right angles with the wall baseline.

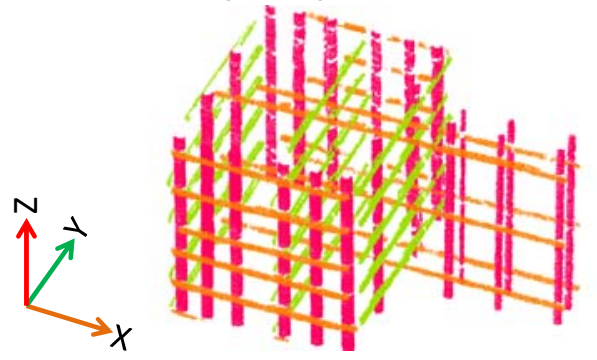

Fig.28 Decomposing point clouds into three pieces

\section{Assembling point clouds into column ties}

The system assembled point clouds into column ties. Figure.29 and Figure.32 show the results of assembling column ties. Furthermore, Figure. 31 shows the result of shape recognition of the point clouds.

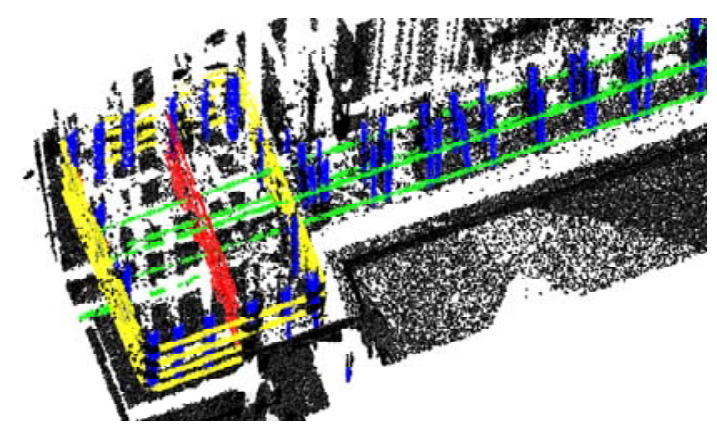

Fig.29 The result of shape recgnition of the point clouds

\section{ConClusions}

The authors scanned reinforcing bars of the columns and the walls at a construction site, then have analyzed the point clouds data using the system described in this paper.

In these experiments, the system was able to identify point clouds of reinforcing bars, and then successfully assemble them into column ties.

The authors have been able to identify the shape of reinforcing bars, and then have been able to count the number of the column ties and the vertical bars. 


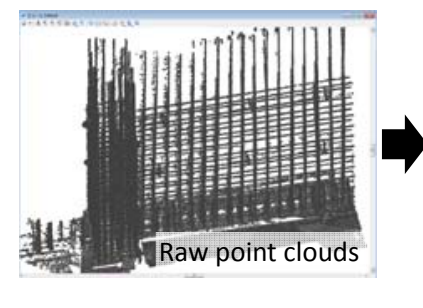

tem Division Symposium, 2009

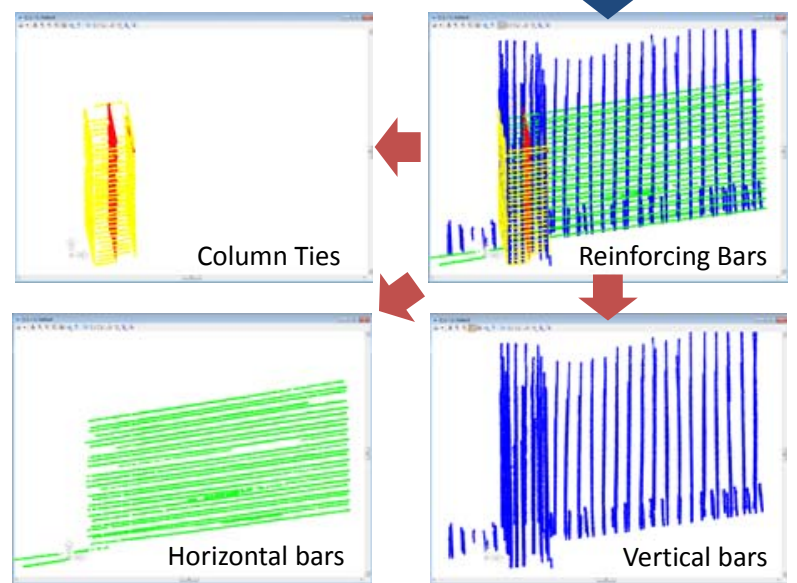

Fig.30 The steps of shape recgnition and abstracing vertical bars, column ties and horizontal bars.

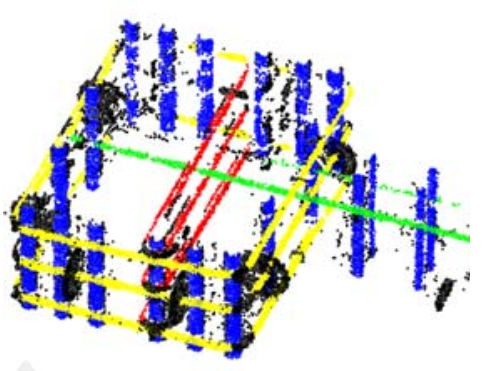

Fig.31 Assembling point clouds into column ties

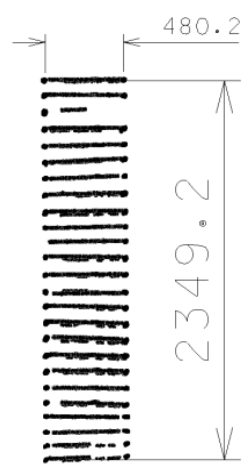

Fig.32 Abstracing column ties

1) The authors developed this system with VBA in MicroStation.

\section{References}

1. Ohstu, S., Sata, T., Mizumoto, M., "Development of Measurement System for the State of rampart using 3-D Laser Profiler", Information Technology Applied in Civil Engineering, Vol.1, 2004

2. Kano, N., Kawahara, Y., Kakizaki, H., Ishioka, H., "Application of 3D Scanner in Building Construction", $26^{\text {th }}$ Building Production Symposium, pp.187-192, July 2009.

3. Adan, A., Xueha, Burcu, Daniel, "Automatic creation of semantically rich $3 \mathrm{D}$ building-models from laser scanner data", The 28th International Symposium on Automation and Robotics in Construction, S10-1, July 2011

4. Lukacs, Marshal, Martin, "Geometric least-squares fitting of spheres, cylinders, cones and tori”, 1997

5. Masuda, H., "An Interactive 3D Modeling System based on Large-Scale Point-Clouds", $19^{\text {th }}$ Japanese Society of Mechanical Engineers, Design and Sys- 